\title{
ORT_17 - DMF-loaded SLN administrated by inhalation route attenuate clinical signs and reduce lung and CNS inflammation in MS animals' model
}

Bárbara Fernandes Pinto ${ }^{1 *}$; Gisela Bevilacqua Rolfsen Ferreira da Silva ${ }^{3}$ : Lucas Kraemer ${ }^{1}$; Gabryella Soares Pinheiro dos Santos ${ }^{1}$; Samantha Ribeiro Bèla ${ }^{1}$; Fábio de Lima Leite ${ }^{2}$; Anselmo Gomes de Oliveira ${ }^{3}$; Alexander Birbrair ${ }^{1}$; Remo Castro Russo ${ }^{1}$; Juliana Carvalho-Tavares ${ }^{1}$.

${ }^{1}$ UFMG - Universidade Federal de Minas Gerais;

${ }^{2}$ Universidade Federal de São Carlos (UFSCAR);

${ }^{3}$ Universidade Estadual Paulista (UNESP).

Introduction: Multiple sclerosis (MS) is a chronic disabling autoimmune disease characterized by inflammatory response, gliosis, demyelination and neuroaxonal degeneration in the central nervous system. Dimethyl fumarate (DMF) has been approved as an oral drug for MS treatment based on its immunomodulatory activities, including neuroprotective and anti-inflammatory effects. However, the drug when administered orally causes serious adverse effects, mainly related to the gastrointestinal system, impairing the patient's adherence to therapy.

Objective: Our objective is to evaluate the possible lung toxicity and the central nervous system effects of inhalation of DMF encapsulated into solid lipids nanoparticles (SLN) in multiple sclerosis animal model, known as experimental autoimmune encephalomyelitis (EAE).

Methodology: EAE was induced with a subcutaneous administration of an emulsion containing MOG3555, Mycobacterium tuberculosis and complete Freund's adjuvant in female C57BL/6J mice. Pertussis toxin (i.p.) was injected at the induction day and 48 hours later. Mice were treated via inhalatory route with DMF-encapsulated nanoparticles (CTRL/SLN/DMF and EAE/SLN/DMF), empty nanoparticles (CTRL/SLN and EAE/SLN) and saline solution (CTRL/saline and EAE/saline) each 72 hours for 21 days. Clinical score, body weight, brain and spinal cord vascular permeability, spinal cord inflammatory infiltration, in vivo leukocyte endothelial interactions, cerebral cytokines levels (IL-10, TNF- $\alpha$, IL-17) and FOXp3 spinal cord level cells were evaluated. To analyzed the lung toxicity due to inhalatory route of administration, the lung vascular permeability, total leucocytes, lung cytokines levels (IL-10, TNF- $\alpha$, IL-17) and the respiratory mechanics (inspiratory capacity and pulmonary resistance) were evaluated.

Results: EAE mice treated with DMF-encapsulated in SLN, when compared to EAE/saline, showed decreased in clinical score and weight loss, reduction in brain and spinal cord vascular permeability and in spinal cord inflammatory cellularity, as well as, an increased in leucocyte rolling and adhesion. It was also observed a decrease in cerebral levels of TNF- $\alpha$ and IL-17 and an increased in FOXp3 cells in spinal cord. At the same time, we observed that EAE animals presented an increased in lung permeability, total leucocytes, TNF- $\alpha$ and IL-17 levels and pulmonary resistance, and a decrease in IL-10 levels and in the inspiratory capacity, while EAE animals treated with DMF encapsulated with SLN were able to revert all these effects.

Conclusion: For the first time, we demonstrated that the lung also was compromitted in EAE disease, suggesting that this organ may be responsible for an essential role in autoimmunity. Moreover, our results also reveal that inhalation of DMF encapsulated in SLN was effective to reduce SNC inflammation, EAE disability progression, and ameliorated lung inflammation and function.

Keywords: Experimental autoimmune encephalomyelitis; DMF-encapsulated in solid lipid nanoparticles; Lung and central nervous system inflammation 